\title{
MULTI-SOURCE REMOTE SENSING IMAGES MATCHING BASED ON IMPROVED KAZE ALGORITHM
}

\author{
WANG Zong-weia, b, WANG Hua-bin ${ }^{\text {b }}$, WANG Guang-hui ${ }^{\text {b }}$, LI Can-hai ${ }^{\text {b }}$, YANG Hua-chao ${ }^{\text {a }}$
}

\author{
a Environment science and Spatial Informatics, China University of Mining and Technology School, Jiangsu Xuzhou 221116, China \\ ${ }^{\mathrm{b}}$ Satellite Surveying and Mapping Application Center, National Administration of Surveying, Mapping and Geoinformation, Beijing \\ 100830, China
}

KEY WORDS: Multi-Source Remote Sensing Images, KAZE, AOS, SIFT, Image Matching, Nonlinear Scale Space, Geometric transformation model

\begin{abstract}
:
SIFT as the representative of the same feature point extraction and matching algorithm has been widely applied in the field of multisource remote sensing image matching. However, it eliminates noise and detects features at different scale levels by building or approximating the Gaussian scale space based on linear. Gaussian blurring does not respect the natural boundaries of objects and smoothes to the same degree details and noise, reducing localization accuracy. To solve this problem, we proposed an improved KAZE algorithm which can build stable nonlinear scale space. Firstly, the extreme points are detected through building stable nonlinear scale space. Secondly, The match result by optimizing the feature points and strictly limiting matching threshold is used to calculate geometric transformation model parameters between two image. Finally, we can use this geometric transformation model to restrict the search space for feature points matching. Experimental results show that the improved KAZE algorithm is significantly better than the before KAZE. Moreover, for detail and texture blurred images, KAZE and its improved algorithm have unique advantages compared to the SIFT.
\end{abstract}

\section{INTRODUCTION}

With the rapid development of remote sensing technology, different sensor resolutions and phase of the multi-source remote sensing images have become an important data source for basic surveying and mapping, agricultural census, meteorological observations, land and resources dynamic monitoring. Due to the influence of external factors such as weather, sunlight, shelter and different imaging time, angle, distance led to the questions of image resolution, pan, rotate, zoom, it has brought great difficulty to multi-source remote sensing images matching work .

SIFT(Scale Invariant Feature Transform) (Lowe, 2004) as the representative of the same feature point extraction and matching algorithm, such as: SURF (H.Bay, 2006), PCA-SIFT (Y.Ke, 2004), ASIFT (Morel, 2009), has been widely applied in the field of multi-source remote sensing images matching. However, it eliminates noise and detects features at different scale levels by building or approximating the Gaussian scale space based on linear. Gaussian blurring does not respect the natural boundaries of objects and smoothes to the same degree both details and noise, reducing localization accuracy and distinctiveness, which causes edge matching poor stability, brings more error matching points and also increases the difficulty of the error matching points elimination. To solve this problem, we proposed an improved KAZE algorithm which can builds stable nonlinear scale space using efficient Additive Operator Splitting (AOS) techniques (Ruan Zong-cai, 2006) and variable conductance diffusion.

\section{REMOTE SENSING IMAGES MATCHING ALGORITHM BASED ON IMPROVED KAZE}

In the first phase of the improved KAZE algorithm, nonlinear scale space is built using efficient Additive Operator Splitting (AOS) techniques and variable conductance diffusion to solve implicit differential equations. And then, feature points are detected by searching for normalized Hessian local maxima at different scales. In the second phase, 64-dimensional double Gaussian weighted M-SURF feature descriptors are constructed. In the third phase, the match result by optimizing the feature points and strictly limiting match threshold is used to calculate geometric transformation model parameters between two images.Finally, That using the geometric transformation model to estimate the matching point's position in the reference image can restrict the search space for feature points matching, which can improve the speed and accuracy of matching. The specific process is shown in Figure 1:

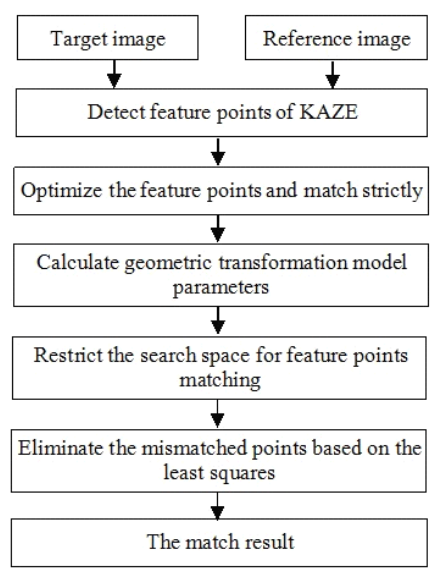

Figure 1. The flow char of the proposed algorithm 


\subsection{Nonlinear Scale Space}

2.1.1 Nonlinear Diffusion Filtering(Pablo, 2012, Zhang Erhua, 2011): Nonlinear diffusion filtering approaches describe the luminance of an image at different scales levels as the divergence of a certain flow function. These approaches are normally described by nonlinear partial differential equations:

$$
\frac{\partial L}{\partial t}=\operatorname{div}(c(x, y, t) \bullet \nabla L)
$$

Where

$$
\begin{aligned}
& L=\text { the luminance of an image } \\
& t=\text { the time of diffusion } \\
& \text { div }=\text { the divergence operators } \\
& \nabla=\text { the divergence operators } \\
& c(x, y, t)=\text { a conductivity function }(c)
\end{aligned}
$$

The function $\mathcal{C}$ is possible to make the diffusion adaptive to the local image structure. The time $t$ is the scale parameter, and larger values lead to simpler image representations. In this paper, the function $\mathcal{C}$ is defined as follows:

$$
c(x, y, t)=g\left(\left|\nabla L_{\sigma}(x, y, t)\right|\right)
$$

where $\nabla L_{\sigma}=$ the gradient of a Gaussian smoothed version of the original image $L_{\sigma}$

The function $g$ is defined as follows:

$$
\begin{aligned}
& g_{1}=\exp \left(-\frac{\left|\nabla L_{\sigma}\right|^{2}}{k^{2}}\right) \quad, \quad g_{2}=\frac{1}{1+\frac{\left|\nabla L_{\sigma}\right|^{2}}{k^{2}}}, \\
& g_{3}=\left\{\begin{array}{cc}
1 & \left|\nabla L_{\sigma}\right|^{2}=0 \\
1-\exp \left(-\frac{3.315}{\left(\left|\nabla L_{\sigma}\right| / k\right)^{8}}\right), & \left|\nabla L_{\sigma}\right|^{2}>0
\end{array}\right.
\end{aligned}
$$

The function $g_{1}$ can promote high-contrast edges. $g_{2}$ can promote wide regions over smaller ones. $g_{3}$ can effectively smooth internal area and retain boundary information. The parameter $k$ is the contrast factor that controls the level of diffusion, which can decide retain how much edge information and large values lead to retain litter edge information .

2.1.2 Building Nonlinear Scale Space: The KAZE takes a similar approach as done in SIFT, discretizing the scale space in logarithmic steps arranged in a series of $O$ octaves and $S$ sub-levels. Unlike SIFT, the KAZE always work with the original image resolution. The octave and sub-level are corresponding to scalar $\sigma$ through the following formula:

$$
\sigma_{i}=(o, S)=\sigma_{0} 2^{\alpha+s / S}, o \in[0 . . O-1], s \in[0 . . S-1], i \in[0 . . M]
$$

$$
\begin{aligned}
& \text { where } \quad \begin{array}{l}
\sigma_{0}=\text { the base scale level } \\
N=O * S=\text { the total number of filtered } \\
\text { images }
\end{array}
\end{aligned}
$$

Because nonlinear diffusion filtering is defined in time terms, the set of discrete scale levels in pixel units is needed to convert to time units. In the case of the Gaussian scale space ,the convolution of an image with a Gaussian of standard deviation $\sigma$ is equivalent to filtering the image for some time $t=\sigma^{2} / 2$. Thus, the mapping relation of transforming the scale space $\sigma_{i}$ to time units is defined as following formula:

$$
t_{i}=\frac{1}{2} \sigma_{i}^{2}, i=\{0 \ldots N\}
$$

$$
\text { Where } \quad t_{i}=\text { the evolution time }
$$

Given an input image, we process image with Gaussian filtering, then calculate image gradient histogram, thus obtain the contrast parameter $k$. According to a group of evolution time, the use of AOS nonlinear scale space algorithm can get all the different scale space images (For example Figure 2):

$$
L^{i+1}=\left(I-\left(t_{i+1}-t_{i}\right) \bullet \sum_{l=1}^{m} A_{l}\left(L^{i}\right)\right)^{-1} L^{i}
$$

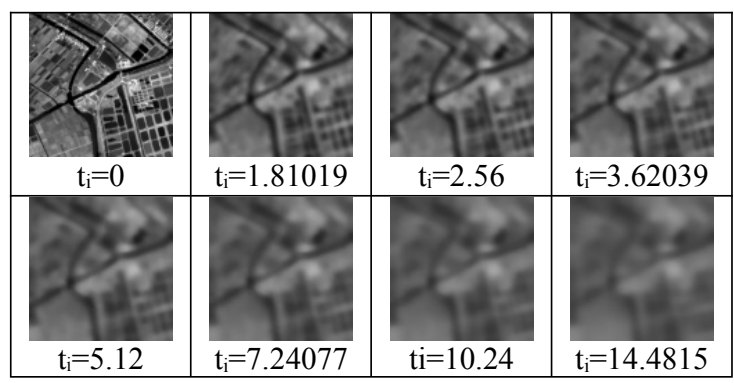

Figure 2. Nonlinear scale space images

\subsection{Feature Points Detection}

KAZE feature points detection is achieved by searching for normalized Hessian local maxima at different scales. The Hessian matrix is calculated as follows: 


$$
L_{\text {Hessian }}=\sigma^{2}\left(L_{x x} L_{x y}-L_{x y}{ }^{2}\right)
$$

Where

$$
\begin{aligned}
& \left(L_{x x}, L_{y y}\right)=\text { the second order horizontal and } \\
& \text { vertical derivatives respectively } \\
& L_{x y}=\text { the second order cross derivative }
\end{aligned}
$$

When searching for maxima, each pixel is compared with all its neighboring points, including its previous and the next scale total of 26 points. When it is larger than its neighboring points of scale and image domain, it is namely maxima points.

After finding the location of feature points, precise positioning reaches to sub-pixel using the method of Lowe proposed in BMVC2002 (Lowe, 2002). This is based on Taylor expansion:

$$
L(X)=L+\left(\frac{\partial L}{\partial X}\right)^{T} X+\frac{1}{2} X^{T} \frac{\partial^{2} L}{\partial X^{2}} X
$$

Sub-pixel coordinates of the feature points are:

$$
\hat{X}=-\left(\frac{\partial^{2} L}{\partial X^{2}}\right)^{-1} \frac{\partial L}{\partial X}
$$

\subsection{Feature Descriptor}

2.3.1 The Dominant Orientation Of Feature Points: To obtain rotation invariant descriptors, it's necessary to estimate the dominant orientation based on the local image structure of feature points. If the feature point scale parameter is $\sigma_{i}$, the search radius is set to $6 \sigma_{i}$. For all the neighboring points on the search circle, first order derivatives $L_{x}$ and $L_{y}$ are weighted with a Gaussian centered at the interest point, so response contribution of which close to the feature points is large and away from the feature points is litter. The derivative responses are represented as points in vector space and the dominant orientation is found by summing the responses within a sliding circle segment covering an angle of $60^{\circ}$. From the longest vector the dominant orientation is obtained.

2.3.2 Building The Feature Descriptor: In this paper, the MSURF descriptor is used to adapt to nonlinear scale space framework. For the scale parameter $\sigma_{i}$ of the feature point, with feature point as the center, the window of $24 \times 24$ is extracted on the gradient image. And the window is divided into $4 \times 4$ sub-regions. The size of each sub-region is $9 \sigma_{i} \times 9 \sigma_{i}$. Neighboring sub-regions have an overlapping band width of $2 \sigma_{i}$. Each sub-region is weighted by a Gaussian kernel $\left(\sigma_{1}=2.5 \sigma_{i}\right)$. And then description Vector of subregion whose length is 4 is calculated.

$$
d_{v}=\left(\sum L_{x}, \sum L_{y}, \sum\left|L_{x}\right|, \sum\left|L_{y}\right|\right)
$$

Afterwards, each sub-region's vector $d_{v}$ is weighted by another size of $4 \times 4$ Gaussian window $\left(\sigma_{2}=2.5 \sigma_{i}\right)$. Finally, through normalized, $4 \times 4 \times 4=64$ dimensional description vector is obtained.

\subsection{Constrained Matching}

The better KAZE feature points are selected based on spatial distribution and information entropy(Yang Hua-chao, 2011). Euclidean distance between eigenvectors of feature descriptors is the similarity measure. Based on Nearest Neighbour Distance Ration(NNDR), optimal matching feature points are obtained by strictly limiting matching threshold $\delta$.

2.4.1 Restrict The Search Space For Feature Points: Matching points obtained by the optimized feature points are used to calculate geometric transformation model parameters. On this basis, we can use the geometric transformation model to estimate the matching point's position in the reference image. And then we can build a circular area whose centre is the position of the estimated points and whose radius is $R$ pixels. If more than one feature point are in the circular area, NNDR is used to search for the best matching point. And only one feature point is in the circular area, the correlation coefficient of template matching is to be used. The method of restricting the search space for feature points matching can get most of the feature points out of the search space and reduce the amount of error matching points caused by "different point homogeneity", which can improve the speed and accuracy of matching.

2.4.2 Eliminating The Mismatched Feature Points: We need to estimate the residual error of each matching point using the least squares principle and statistic its Root Mean Square Error(RMSE). If the RMSE is greater than threshold $\gamma$, the matching point whose RMSE is the greatest, is eliminated until the RMSE is less than $\gamma$. The RMSE is calculated as follows:

$$
R M S E=\sqrt{\frac{\sum_{1}^{N}\left(\Delta x^{2}+\Delta y^{2}\right)}{N}}
$$

Where

$$
\begin{aligned}
& \Delta x=\text { the residual error in } x \text { direction } \\
& \Delta y=\text { the residual error in } y \text { direction } \\
& N=\text { the amount of matching points }
\end{aligned}
$$

\section{EXPERIMENT AND RESULT ANALYSIS}

In this paper, we select multi-source remote sensing image of "ZY-3", SPOT5 and IRS-P5 and make a comprehensive analysis between the improved KAZE algorithm with SIFT and KAZE. Random Sample Consensus (RANSAC) is used to eliminate mismatched points in SIFT and KAZE. Under Window 7 operating system, Visual Studio 2010 and OpenCV(Open Computer Vision Library) environment are 
integrated to write the algorithm experiment program. The main parameters of hardware environment is that: CPU is Intel(R) Core (TM) i3-2310M 2.10Ghz. RAM is $2 \mathrm{G}$.

In order to make the table and figure seemed brief, another form of shorthand is used in the following tables and figures:

Where $\quad$ KAZE $+=$ the improved KAZE

$\mathrm{t} 1=$ the time of detecting feature points

$\mathrm{t} 2=$ the time of matching

$\mathrm{t} 3=$ the total time

num $=$ the amount of right matching points

\begin{tabular}{|c|c|c|c|c|}
\hline Algorithm & $\mathrm{t} 1 / \mathrm{s}$ & $\mathrm{t} 2 / \mathrm{s}$ & $\mathrm{t} 3 / \mathrm{s}$ & num \\
\hline SIFT & 0.962 & 1.295 & 2.257 & 104 \\
\hline KAZE & 1.006 & 0.437 & 1.443 & 23 \\
\hline KAZE+ & 1.006 & 0.311 & 1.317 & 49 \\
\hline
\end{tabular}

Table 1 . The matching result of ZY-3 stereo images $(509 * 512)$

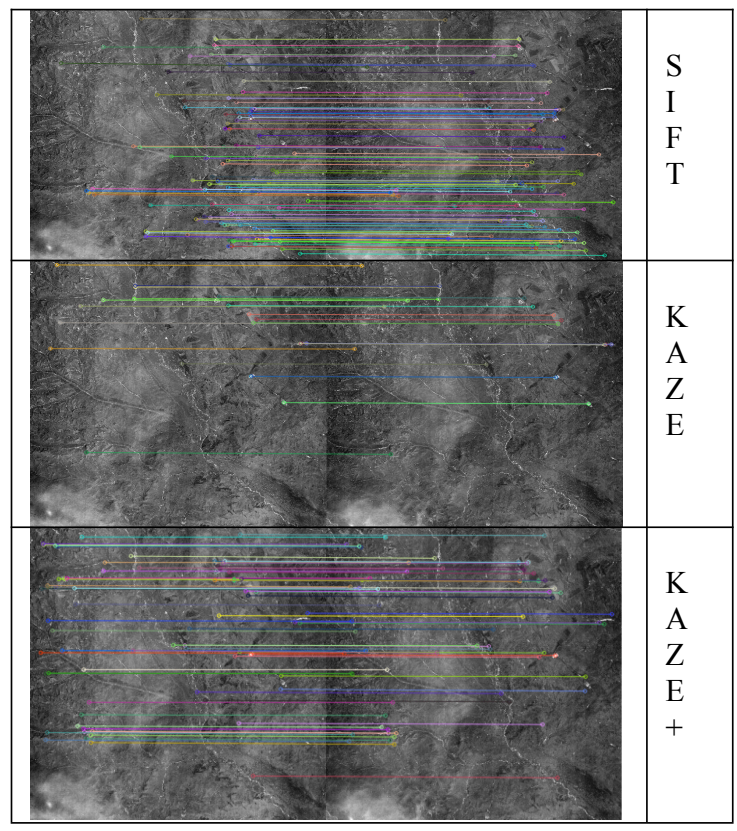

Figure 3. The matching result of ZY-3 stereo images(509*512)

\begin{tabular}{|c|c|c|c|c|}
\hline Algorithm & $\mathrm{t} 1 / \mathrm{s}$ & $\mathrm{t} 2 / \mathrm{s}$ & $\mathrm{t} 3 / \mathrm{s}$ & num \\
\hline SIFT & 0.771 & 1.445 & 2.216 & 0 \\
\hline KAZE & 0.803 & 0.361 & 1.164 & 8 \\
\hline KAZE+ & 0.803 & 0.235 & 1.038 & 21 \\
\hline
\end{tabular}

Table 2. The matching result of IRS-P5 image $(434 * 379)$ and SPOT5 image $(246 * 225)$

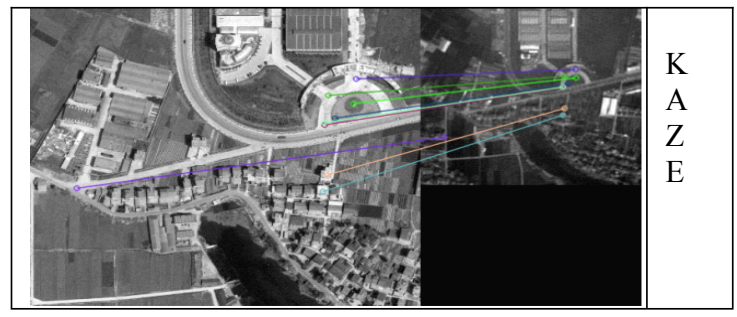

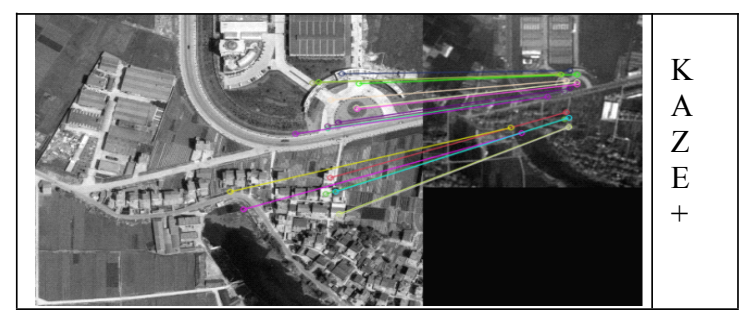

Figure 4. The matching result of IRS-P5 image (434*379) and SPOT5 image $(246 * 225)$

\begin{tabular}{|c|c|c|c|c|}
\hline Algorithm & $\mathrm{t} 1 / \mathrm{s}$ & $\mathrm{t} 2 / \mathrm{s}$ & $\mathrm{t} 3 / \mathrm{s}$ & num \\
\hline SIFT & 0.639 & 1.910 & 2.549 & 0 \\
\hline KAZE & 0.754 & 0.807 & 1.561 & 57 \\
\hline KAZE+ & 0.754 & 0.567 & 1.321 & 92 \\
\hline
\end{tabular}

Table 3. The matching result of SPOT5 images $(509 * 512)$ before and after Wenchuan earthquake

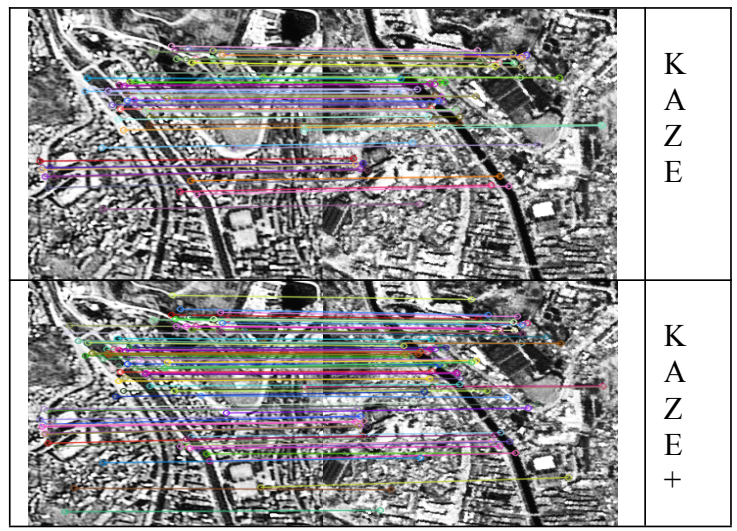

Figure 5. The matching result of SPOT5 images $(509 * 512)$ before and after Wenchuan earthquake

According to the table 1 to 3 , for the same image, the feature points detection time of the three algorithms is almost the same. From the table 2 to 3 and figure 4 to 5, for multi-source remote sensing images of larger difference resolution and phase, the amount of matching points of KAZE is significantly better than SIFT. The time and the amount of matching points of the improved KAZE are significantly better than the before KAZE. Especially for the remote sensing images before and after an earthquake, the KAZE and the improved KAZE show their unique advantages by building non-linear scale space which is no loss of details.

\section{CONCLUSION}

The paper aims at that SIFT builds linear scale space, easily to cause detail losed and boundary blurred. On the base of KAZE that can build nonlinear scale space, the improved KAZE through optimizing the feature points to calculate geometric transformation model parameters and restricting the search space to match feature points, is significantly better than KAZE. Its matching time is shorter and it has more right matching points The improved KAZE is preferentially selected as the matching algorithm for detail and texture blurred multi-source remote sensing images. 
International Archives of the Photogrammetry, Remote Sensing and Spatial Information Sciences, Volume XL-7/W1, 3rd ISPRS IWIDF 2013, 20 - 22 August 2013, Antu, Jilin Province, PR China

\section{REFERENCES}

Lowe D G., 2004. Distinctive image features from scaleinvariant key points. International Journal of Computer Vision, 60(2), pp. 91-110.

Bay H.,2008. SURF:Speeded up Robust Features. Computer Vision and Image Understanding, 110(3), pp. 346-359.

Ke Y., 2004. PCA-SIFT:A More Distinctive Representation for Local Image Descriptors. IEEE Conference Computer Vision and Pattern Recognition, pp. 506-513.

J.M. Morel.,2009. ASIFT:A New Framework for Fully Affine Invariant Image Comparison. SIAM Journal on Imaging Sciences, pp. 438-469.

Ruan Zong-cai., 2006. AOS based seeded linear diffusion algorithm for image segmentation. Microelectronics \& Computer, 23(12), pp. 89-95.

Pablo F. Alcantarilla., 2012. KAZE Features, ECCV, Florence, Italy.

Zhang Er-hua., 2011. Non-linear anisotropic diffusion filtering for 3D seismic noise removal and structure enhancement. Progress in geophysics, 25(3), pp. 866-870.

Lowe D G.,2002. Invariant features from interest point groups. In: British Machine Vision Conf. (BMVC), Cardiff, UK .

Yang Hua-chao., 2011. Dense matching for wide base-line stereo images based on SIFT. Acta Geodaetica et Cartographica Sinica, 40(5), pp. 537-543. 